\title{
DISTAL SCAPHOID RESECTION ARTHROPLASTY FOR SCAPHOID NONUNION WITH RADIOSCAPHOID ARTHRITIS
}

\author{
Pavel Drac ${ }^{a *}$, Pavel Manak ${ }^{\mathrm{a}}$, Lucie Pieranova ${ }^{\mathrm{b}}$
}

\author{
${ }^{a}$ Department of Traumatology, University Hospital, Olomouc, Czech Republic \\ ${ }^{b}$ Clinic of Radiology, University Hospital, Olomouc \\ e-mail:dracpa@fnol.cz
}

Received: December 1, 2006; Accepted: February 5, 2006

Key words: Scaphoid nonunion/Radioscaphoid arthritis/Distal scaphoid resection arthroplasty

The authors compare the functional outcome of 8 patients treated by distal scaphoid resection arthroplasty for scaphoid nonunion with symptomatic wrist arthritis before surgery and after a minimum follow-up of 6 months. There was a significantly better range of radial deviation and grip strength at the time of re-examination. Significantly fewer patients complained of resting pain. By contrast the change of radiolunate angle measured before the surgery and at the time of re-examination was statistically insignificant. Our preliminary results show that distal scaphoid resection arthroplasty seems to be a useful treatment method of scaphoid nonunion with symptomatic wrist arthritis.

\section{INTRODUCTION}

The treatment of long-standing scaphoid nonunion associated with symptomatic wrist arthritis remains a challenging problem. Treatment possibilities include proximal row carpectomy, scaphoid excision with limited intercarpal arthrodesis and wrist arthrodesis ${ }^{1-3}$.

In contrast to these methods, distal scaphoid resection arthroplasty is a simply performed and motion-preserving procedure without risk of nonunion and with a short period of postoperative immobilization.

The purpose of this study was to evaluate the results of distal scaphoid resection arthroplasty after a minimum follow-up of 6 months.

\section{MATERIAL AND METHODS}

From October 2003 to April 2005 eight patients with scaphoid nonunion and symptomatic radioscaphoid arthritis were treated by distal scaphoid resection arthroplasty at the Department of Traumatology of the Teaching Hospital in Olomouc. There were one woman and seven men with average age 37 (24-57) years. The average time from the initial injury to surgery was 57 (17-240) months. Three patients underwent previous surgery (osteosynthesis with Matti-Russe, iliac bone graft or vascularized bone graft). Five patients had not been surgically treated before. Radioscaphoid arthritis was found on the preoperative radiographs of all eight patients.

The distal scaphoid resection arthroplasty was completed with limited radial styloidectomy (done in three of the eight wrists) and excision of posterior interosseus antebrachial nerve (performed in last five consecutive patients).
The minimum follow-up was six (6-24) months after the distal scaphoid resection arthroplasty.

We compared the flexion-extension arch, palmar flexion, dorsal flexion, radial and ulnar deviations, grip strength, radiolunate angle, resting pain and pain during physical activities before surgery and during re-examination. The flexion-extension arch was measured in degrees. Radiographs were evaluated for changes in radiolunate angle. The flexions, deviations and grip strength (using a pneumatic dynamometer) were compared with the opposite, unaffected wrist.

The differences between the results evaluated before the surgery and during the re-examinations were tested by the Student's t-test, the Wilcoxon signed rank test and the Chi-square test. The results were considered to be significant if $\mathrm{p}<0.05$.

\section{Surgical technique of distal scaphoid resection arthroplasty:}

All operations were performed under general anaesthesia with a pneumatic tourniquet applied to the upper arm and insufflated during the surgery. A 3-4 cm wave-shaped incision was made palmarly starting from the scaphoid tuberosity proximally over the flexor carpi radialis (FCR) tendon. The sheath of the the FCR was opened longitudinally and the tendon was retracted radially. Surgical exposure was extended distally from the STT joint to the scapholunate articulation proximally. The capsule was incised longitudinally. Subperiostal dissection was carried out to preserve the dorsal intercarpal ligaments. A $1.5 \mathrm{~mm}$ K-wire was utilized as a joy-stick in some cases. The distal fragment was removed en bloc. With the aid of image intensifier wrist stability and a contingent residual impingement throughout the entire range of motion were assessed. If radial deviation was found to be 
limited intraoperatively, a limited radial styloidectomy was performed.

Excision of posterior interosseus antebrachial nerve was performed using $5 \mathrm{~cm}$ skin incision starting $3 \mathrm{~cm}$ proximally to the distal radioulnar joint. After oppening the antebrachial fascia and retraction of extensor digitorum communis and extensor indicis proprius the nerve was found and 10-15 mm excision was performed.

The procedure was completed by repairing the capsule, radio-scapho-capitate ligament, soft tissue closure and skin suture. The patients were immobilized in a shortarm thumb spica splint for 2-3 weeks, after which physiotherapy was begun.

\section{RESULTS}

The average length of the follow-up period was 10 months (range 6-24 months). The changes of flexionextension arch, palmar flexion and dorsal flexion, ulnar deviation, radiolunate angle and pain during physical activitiy were statistically insignificant. The change in dorsal flexion was of borderline significance $(p=0.050)$. Using Wilcoxon signed rank test the change was statistically insignificant however $(p=0.063)$. The radial deviation, grip strength and the resting pain improved and the changes were statistically significant $(p<0.05)$. We did not find newly developed degenerative arthritis at the midcarpal joint in any patient.

\section{DISCUSSION}

The ideal salvage procedure for long-standing scaphoid nonunion associated with symptomatic arthritis is to pro- vide a pain-free, stable wrist with minimal loss of motion and it should be technically simple to perform ${ }^{4}$.

Excision of the carpal scaphoid for treatment of nonunion was reported as early as 1905 by Codman and Chase (ref. ${ }^{5}$ ). Once the scaphoid was excised, however, substantial carpal destabilization occurred. Proximoradial migration of the capitate leads to progress to carpal collapse. The long-term outcome of excision of the proximal portion of an nonunited scaphoid fracture is similar to that for total scaphoidectomy because the capitate was also left devoid of one of its proximal supports ${ }^{6}$. And, as published by Dwyer ${ }^{7}$ in 1949 “...arthritic changes in cases of scaphoid nonunion usually appear first between the radial styloid and distal scaphoid fragment. It seems irrational therefore to retain the distal fragment".

The using of distal scaphoid resection arthroplasty for decompression of the radial side of the wrist to retain the radiocarpal joint and wrist stability, while eliminating current degenerative changes, for scaphoid nonunion was published by Ruch ${ }^{4}$ in 1998 and subsequently by Malerich ${ }^{8}$ in 1999. Theoretically, excision of the distal fragment eliminates the impingement symptoms caused by motion at the nonunion site and between the radial styloid and distal scaphoid ${ }^{5}$. The main advantages of this method are that this is a simply performed and motion-preserving procedure without risk of nonunion and with a short period of postoperative immobilization. Furthermore, if wrist instability appears as a consequence of the loss of the distal portion of the bone, midcarpal fusion still can be used to solve the problem ${ }^{6}$.

Our operative indication was scaphoid nonunion with symptomatic radioscaphoid arthritis without involvement of the lunocapitate joint. The same indication was presented by Ruch ${ }^{4}$ and Malerich ${ }^{8}$ in their studies. In-Ho ${ }^{5}$ in 2003, however, also used this method to treat two patients

Table 1. Results evaluated before the surgery, during re-examination (after a minimum follow-up of 6 months) and statistical analysis. The differences were tested by Student's t-test, the Wilcoxon signed rank test $\left({ }^{*}\right)$ and the Chi-square test $(* *)$.

\begin{tabular}{|l|c|c|c|}
\hline & Before surgery & During re-examination & Statistical analysis \\
\hline Flexion-extension arch & $\mathrm{n}=8$ & $\mathrm{n}=8$ & $\mathrm{P}=0.175$ \\
\hline Palmar flexion & $101.4^{\circ} \pm 12.1^{\circ}$ & $72.7 \pm 18.4$ & $\mathrm{P}=0.894$ \\
\hline Dorsal flexion & $68.1 \pm 15.5$ & $82.8 \pm 13.6$ & $\mathrm{P}=0.050\left(^{*} 0.063\right)$ \\
\hline Radial deviation & $77.9 \pm 9.9$ & $84.8 \pm 30.2$ & $\mathrm{P}=0.013$ \\
\hline Ulnar deviation & $56.5 \pm 20.5$ & $66.5 \pm 17.7$ & $\mathrm{P}=0.717$ \\
\hline Grip strength (\%) & $68.1 \pm 18.7$ & $79.9 \pm 18.6$ & $\mathrm{P}=0.001$ \\
\hline Radiolunate angle & $60.8 \pm 12.2$ & $-22.8^{\circ} \pm 9.6^{\circ}$ & $\mathrm{P}=0.289$ \\
\hline Resting pain & $-22.0^{\circ} \pm 11.0^{\circ}$ & 0 & $\mathbf{P}=\mathbf{0 . 0 0 7 * *}$ \\
\hline $\begin{array}{l}\text { Pain during physical } \\
\text { activities }\end{array}$ & 6 & 4 & $\mathrm{P}=0.077^{* *}$ \\
\hline
\end{tabular}


with arthritic changes in the midcarpal joint and who experienced good pain relief and range of motion. Soejima ${ }^{9}$ reported the presence of capitolunate arthritis in six to nine operated patients.

In our study we found improvement of the flexion-extension arc, palmar flexion, dorsal flexion and radial deviation at the time of re-examination but only the change of radial deviation was statistically significant. Malerich ${ }^{8}$ presented in the group of 19 patients improvement of flexion extension arc (from $49^{\circ}$ to $92^{\circ}$ ) and radio-ulnar deviation (from $23^{\circ}$ to $41^{\circ}$ ) but without statistical analysis. Soejima ${ }^{9}$ reported on 9 patients with improvement of flexion-extension arc, which was statistically significant $(\mathrm{p}=0.001)$. In-Ho ${ }^{5}$ found in a group of 7 patients improvement in flexion-extension arc from $81.4^{\circ}$ to $120^{\circ}$ and radial-ulnar deviation improved from $30^{\circ}$ to $45^{\circ}$.

Assessing the grip strength we found a statistically significant improvement from $60.8 \%$ to $79.9 \%$ of the opposite, unaffected wrist after the surgery. Soejima ${ }^{9}$ described a change from $40 \%$ to $77 \%$ and Mallerich ${ }^{8}$ reported postoperative improvement to $75 \%$ of the opposite wrist.

To assess the progression of carpal collapse we measured and compared the radiolunate angle on lateral X-ray of the wrist before the surgery and during the re-examination. The change was statistically insignificant. Soejima ${ }^{9}$ presented a similar outcome - change in radiolunate angle from $-26^{\circ}$ to $-27^{\circ}$ (also statistically insignificant) and Malerich ${ }^{8}$ who found a postoperative change of radiolunate angle of only $2^{\circ}$. By contrast the change of radiolunate angle measured by $\mathrm{In}-\mathrm{Ho}^{5}$ was from $-13.6^{\circ}$ to $-25.7^{\circ}$ but without statistical analysis.

All of our eight patients were free of persistent pain during the re-examination $(p=0.007)$ and a half of them were free of pain during physical activity. Analogically, Soejima ${ }^{9}$ reported on four of nine patients free of persistent pain and Malerich $^{8}$ presented thirteen patients of nineteen who had complete pain relief.

We found no newly developed degenerative arthritis at the midcarpal joint in any patient. Malerich ${ }^{8}$ reported on three $(16 \%)$ patients with progressive degenerative changes and Soejima ${ }^{9}$ found new arthritis at the proximal scapholunate capitate articulation in one of nine patients. However, the average follow-up of both studies was longer than ours - 49 respectively 28.6 months. This was, along with a small sample, the main limitation of our study.

\section{CONCLUSION}

The functional and X-ray outcome in patients with scaphoid nonunion and symptomatic radioscaphoid arthritis treated by distal scaphoid resection arthroplasty after an average follow-up of 10 months after the surgery are hopeful. Longer follow-up and a larger sample will be necessary to evaluate the real contribution of this method.

\section{REFERENCES}

1. Jebson JL, Hayes EP, Engber WD. (2003) Proximal row carpectomy: A minimum 10-year followw-up study. J Hand Surg $28 \mathrm{~A}$, 561-569.

2. Kirchenbaum D, Schneider LH, Kirkpatrick H, Adams DC, Cody RP. (1993) Scaphoid excision and capitolunate arthrodesis for radioscaphoid arthritis. J Hand Surg 18A, 780-785.

3. Tomanio MM, Miller RJ, Cole I, Burton RI. (1994) Scapholunate advanced collapse wrist: proximal row carpectomy or limited wrist arthrodesis with scaphoid excision? J Hand surg 19A, 134-141.

4. Ruch DS, Chang DS, Yang CC. (2001) Arthroscopic evaluation and treatment of scaphoid nonunion. Hand Clinics 17, 655-662.

5. In-Ho J, Chang-Wug O, Byung-Chul P, Joo-Chul I, Poong-Taek K. (2003) Advanced wrist arthritis due to scaphoid non-union. SICOT Online Report E044.

6. Garcia-Elias M, Lluch A. (2001) Partial excision of scaphoid: is it ever indicated? Hand Clinics 17, 687-695.

7. Dwyer FC. (1949) Excision of the carpal scaphoid for ununited fracture. J Bone Joint Surg 31B, 572-577.

8. Malerich MM, Clifford J, Eaton B, Eaton R, Littler W. (1999) Distal scaphoid resection arthroplasty for the treatment of degenerative arthritis secondary to scaphoid nonunion. J Hand Surg $24 A$, 1196-1205.

9. Soejima O, Iida H, Hanamura T, Naito M. (2003) Resection of the distal pole of the scaphoid for scaphoid nonunion with radioscaphoid and intercarpal arthritis. J Hand Surg 28A, 591-596. 